\title{
Anais do II Simpósio de Reabilitação Esportiva - SIMREABESP
}

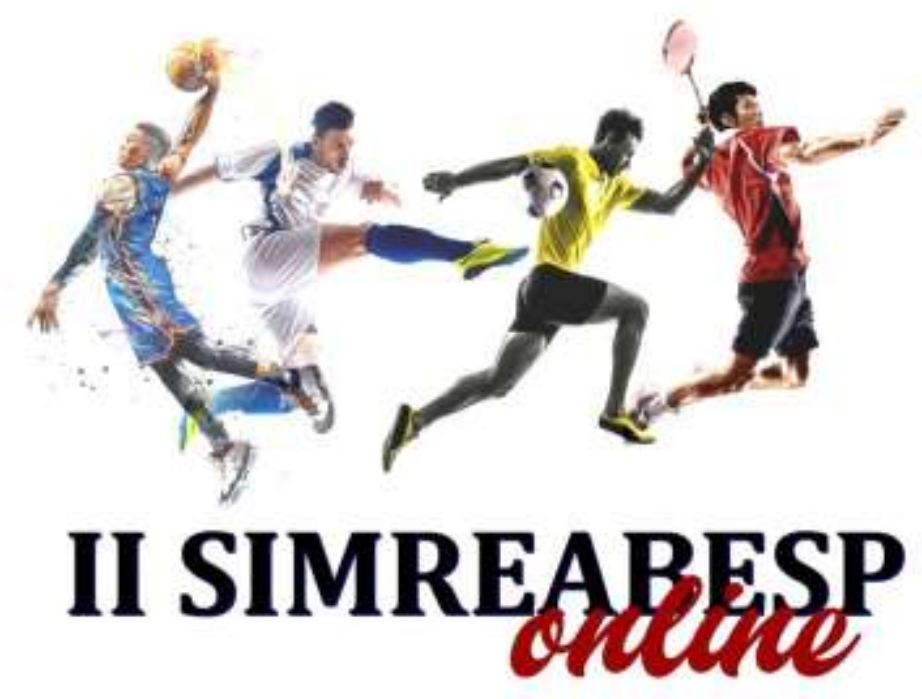

Apoio:

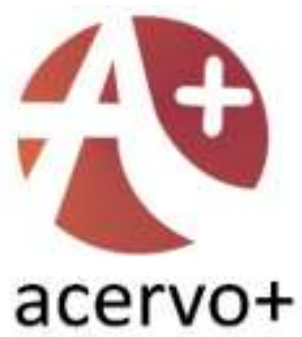

Eventos

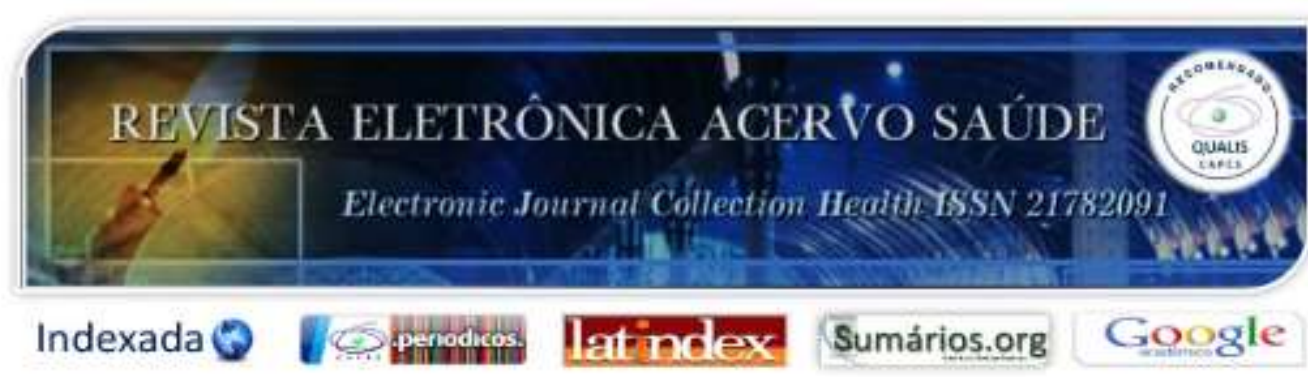




\section{SUMÁRIO}

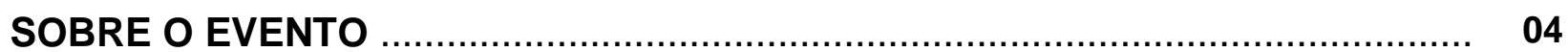

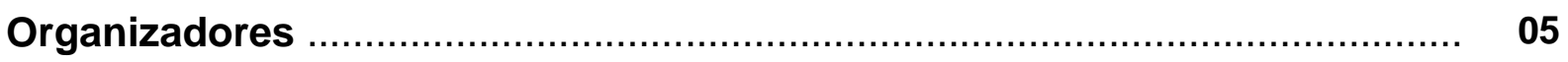

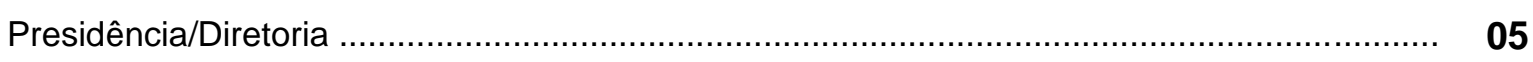

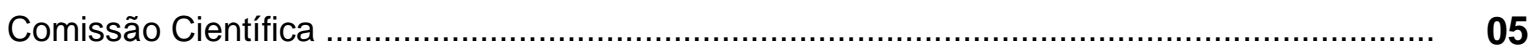

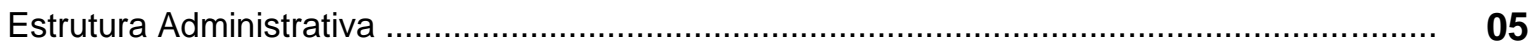

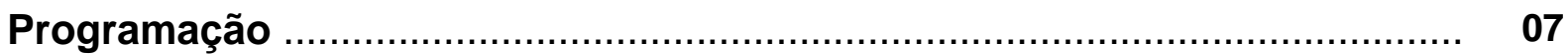

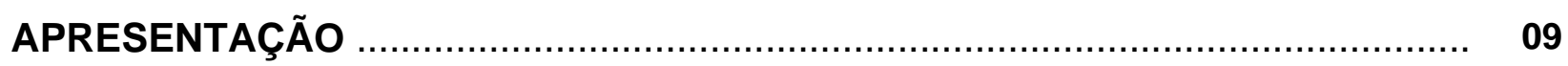

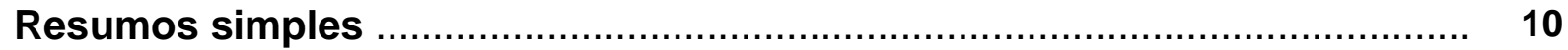

Consumo alimentar em praticantes de crossfit: Uma revisão integrativa......................... $\quad \mathbf{1 0}$

Gisele Viana Moura, Mateus Cunha de Sousa, Palloma de Sousa, Andrea Nunes Mendes de Brito

Etiopatogenia da morte súbita cardíaca em jovens atletas

André Rodrigues Carvalho, Danyele Holanda da Silva, Izabelle Macedo de Sousa

Influência da pandemia do COVID-19 sobre o ganho de peso da população: Uma revisão integrativa

Gisele Viana Moura, Andressa Kelly Lima do Nascimento, Mateus Cunha de Sousa, Carlos Henrique Ribeiro Lima

O treinamento de equilíbrio como estratégia de controle postural em atletas com instabilidade crônica do tornozelo.

Bárbara Leite da Silva, Danyele Holanda da Silva, Rita de Cássia Mendes de Sousa Janaína de Moraes Silva

Suporte nutricional em atletas lesionados: Uma Revisão Integrativa.

Gisele Viana Moura, Mateus Cunha de Sousa, Carlos Henrique Ribeiro Lima, Matheus

Willian Ribeiro de Sousa

Efeitos da Eletroestimulação de Corpo Inteiro no metabolismo, na composição corporal e no desempenho físico.

Daniel dos Santos Nunes, Samuel Nunes Morais, Kelson Nonato Gomes da Silva Incidência de lesões musculoesqueléticas em praticantes de crossfit: revisão sistemática.

Talyta da Silva Guimarães, Jederson Valentim Silva, Ariane Luz Carvalho Influência da Vitamina D sobre a massa óssea em indivíduos fisicamente ativos.

Gisele Viana Moura, Rayana Rodrigues da Silva, Tatiele Castelo de Oliveira, Vanessa Batista de Sousa Lima 
Atuação da fisioterapia no pós-operatório de reconstrução do ligamento cruzado anterior (LCA).

Jessé Sales de Barros, Luiz Valdean Sobrinho Nascimento, Mariana Andrade Jaques de Castro, Haértori da Silva Leal

Dados antropométricos e ingestão alimentar em atletas de voleibol: Uma revisão Integrativa

Mateus Cunha de Sousa, Gisele Viana de Moura, Jerônimo Gregrio Da Silva Neto, Andrea Nunes Mendes de Brito

Efeitos da acupuntura em atletas: revisão bibliográfica.

Danyele Holanda da Silva, Letícia de Sousa Vidal. Bárbara Leite da Silva' Rita de Cássia Mendes de Sousa, Janaína de Moraes Silva

Efeitos das meias compressivas em atletas: uma revisão bibliográfica.

Danyele Holanda da Silva, Letícia de Sousa Vidal, André Rodrigues Carvalho, Neivaldo

Ramos da Silva, Izabelle Macedo de Sousa

Uso da eletroestimulação em pós-operatório de cirurgia de reconstrução do ligamento cruzado anterior em atletas: revisão bibliográfica

Rita de Cássia Mendes de Sousa, Danyele Holanda da Silva, Bárbara Leite da Silva, Janaína de Moraes Silva

AGRADECIMENTOS 


\section{SOBRE O EVENTO}

A prática baseada em evidências tem surgido como um movimento que possibilita a melhoria da qualidade da assistência à saúde por meio da integração entre a experiência individual e a melhor evidência científica disponível proveniente de investigação. Contudo, apesar do crescimento exponencial do conhecimento - a par da disponibilidade à informação através das plataformas digitais - o uso e aplicação de pesquisas como base para a tomada de decisões na prática clínica ainda tem sido um desafio, inclusive no contexto da reabilitação esportiva.

O Simpósio de Reabilitação Esportiva - SIMREABESP surgiu diante da necessidade de se repensar o cuidado prestado aos indivíduos envolvidos no desporto partindo de uma visão multiprofissional e embasada na prática baseada em evidências. O objetivo do evento tem sido proporcionar o desenvolvimento acadêmico e profissional no âmbito esportivo, estimulando a participação dos profissionais de Fisioterapia, Educação Física e áreas afins em atividades de pesquisa e produção científica, visando complementar sua formação e enriquecer seus conhecimentos na área do desporto.

Em sua segunda edição realizada entre os dias 30 e 31 de outubro de 2020, o evento contou com a participação de palestrantes renomados de diversas regiões do país que compartilharam as suas experiências clínicas bem como as melhores evidências científicas na área da reabilitação esportiva. Além da realização de rodas de conversa e apresentação de trabalhos científicos, reforçando o papel do evento como um espaço de encontro e troca de experiências entre acadêmicos e profissionais.

Devido à pandemia pelo novo coronavírus - COVID 19 e às restrições sanitárias decorrentes desta, o Simpósio ocorreu de forma totalmente online pela plataforma de videoconferência Zoom. As palestras, mesas redondas e apresentações de trabalhos foram realizadas ao vivo e os palestrantes disponibilizaram de ferramentas variadas para sanar as dúvidas dos participantes a fim de evitar prejuízos às interações necessárias para a troca de saberes.

JOÃO PAULO CASTRO SOARES

Diretor do II SIMREAESP

ANDRÉ RODRIGUES CARVALHO

Diretor Científico do II SIMREABESP 


\section{Organizadores do Evento}

\section{Presidência/Diretoria}

Coordenador Docente do Evento

Prof. Dr. Francisco Valmor Macedo Cunha

Diretoria do Evento

João Paulo Castro Soares

Andrei lago Viana Soares Feitosa

\section{Comissão Científica}

Coordenadora Docente da Comissão Científica

Profa. Ma. Nidiany da Silva Medeiros Silva

Diretoria Científica

André Rodrigues Carvalho

Mylena Cardoso Sales

Integrantes da banca avaliadora

Alessandro Carvalho Alves dos Santos

Bruno Viana Rosa

Eduardo Henrique de Moura

Hivana Mouta Gomes de Sousa

José Wennas Alves Bezerra

Marcos Vinicio Lopes Barros

\section{Estrutura Administrativa}

Comunicação

Fernanda Eloi Oliveira Fernandes 
Mayara Martins de Melo

Nyelle de Sousa Moura

\section{Finanças}

Carlos Eduardo Nunes Vieira

\section{Palestras}

Amanda Oliveira Leão

Janayra Castro da Silva

Neivaldo Ramos da Silva

Victor Hugo Santos Natur

\section{Patrocínio}

Carolina Vieira Siena Martins

Ingrid da Silva Melo

Lia de Sousa Pádua

Mateus Costa Silva

Rumana de França Almeida 


\section{Programação}

\begin{tabular}{|c|c|l|c|c|}
\hline \multicolumn{5}{|c|}{ Dia 1-30/10/2020 } \\
\hline Horário & Atividade & \multicolumn{1}{|c|}{ Tema } & Palestrantes & Local \\
\hline 08:00- 09:30 & $\begin{array}{c}\text { Mesa } \\
\text { Redonda }\end{array}$ & $\begin{array}{l}\text { Cirurgia e Reabilitação do } \\
\text { LCP }\end{array}$ & $\begin{array}{l}\text { Bruno Machado e } \\
\text { Riccardo Albano }\end{array}$ & Online \\
\hline 09:30-11:00 & Palestra & $\begin{array}{l}\text { Concussão em crianças } \\
\text { menores de 12 anos em } \\
\text { clubes e escolinhas de } \\
\text { futebol: Pazareno Júnior } \\
\text { tratamento, prognóstiça e } \\
\text { as principais } \\
\text { recomendações das }\end{array}$ & Online \\
confederações de futebol \\
pelo mundo. \\
11:00-12:00
\end{tabular}




\begin{tabular}{|c|c|c|c|c|}
\hline & & $\begin{array}{l}\text { pessoas acima do peso } \\
\text { conseguirem resultados } \\
\text { definitivos? Visão integrada } \\
\text { de um profissional de } \\
\text { educação física e uma } \\
\text { nutricionista. }\end{array}$ & & \\
\hline 09:00 - 10:00 & $\begin{array}{l}\text { Mesa } \\
\text { redonda }\end{array}$ & $\begin{array}{l}\text { Reabilitação de coluna: Um } \\
\text { olhar multidisciplinar }\end{array}$ & $\begin{array}{l}\text { Bruno Rosa e } \\
\text { Eduardo Moura }\end{array}$ & Online \\
\hline \multicolumn{5}{|c|}{ Intervalo } \\
\hline $11: 00-12: 00$ & $\begin{array}{l}\text { Mesa } \\
\text { redonda }\end{array}$ & $\begin{array}{l}\text { Reabilitação das principais } \\
\text { lesões no crossfit }\end{array}$ & $\begin{array}{l}\text { Kelson Gomes e } \\
\text { Helder Mourão }\end{array}$ & Online \\
\hline 12:00 - 13:00 & \multicolumn{3}{|c|}{ Apresentação de trabalhos científicos } & Online \\
\hline $14: 00-15: 00$ & Palestra & $\begin{array}{l}\text { Psicologia do esporte e } \\
\text { ferramentas terapêuticas }\end{array}$ & Rodrigo Almeida & Online \\
\hline $15: 00-16: 00$ & Palestra & $\begin{array}{l}\text { Retreinamento das lesões } \\
\text { de joelho, aspectos } \\
\text { biopsicossociais }\end{array}$ & Marcelo Bezerra & Online \\
\hline $16: 00-17: 00$ & Palestra & $\begin{array}{lr}\text { Periodização } & \text { no } \\
\text { fisiculturismo: } & \text { como } \\
\text { periodizar o treino de } \\
\text { acordo com a categoria, e } \\
\text { como diminuir o risco de } \\
\text { lesões }\end{array}$ & Eudes Silveira & Online \\
\hline $17: 00-18: 00$ & Palestra & $\begin{array}{l}\text { O impacto da Nutrição na } \\
\text { reabilitação do idoso } \\
\text { desportista }\end{array}$ & Eudilene Rufino & Online \\
\hline 18:00 - 19:00 & $\begin{array}{l}\text { Mesa } \\
\text { redonda }\end{array}$ & $\begin{array}{l}\text { Atuação transdisciplinar no } \\
\text { futebol: } \quad \text { Reabilitação, } \\
\text { prevenção e desempenho }\end{array}$ & $\begin{array}{l}\text { Paulo Adriano } \\
\text { Schwingel e } \\
\text { Ângelo Raposo }\end{array}$ & Online \\
\hline \multicolumn{5}{|c|}{ Intervalo } \\
\hline $20: 00-21: 00$ & Palestra & Dor lombar em atletas & Eduardo Ferreira & Online \\
\hline $21: 00-22: 00$ & Palestra & $\begin{array}{l}\text { Tenho Hérnia de disco, } \\
\text { posso correr? }\end{array}$ & Marcos Vinicio & Online \\
\hline
\end{tabular}




\section{APRESENTAÇÃO}

Consolidando a missão do evento de disseminar o conhecimento científico na área da reabilitação esportiva, o II SIMREABESP possibilitou a submissão, avaliação e apresentação de resumos simples na forma de estudos de caso, relatos de experiência, estudos originais e revisões de literatura nas diversas áreas do conhecimento em saúde.

O documento de Anais do II SIMREABESP traz 13 revisões de literatura selecionadas após avaliação realizada de forma imparcial por profissionais atuantes na área da reabilitação esportiva e envolvidos com a pesquisa e docência, respeitando as normas metodológicas da Revista Eletrônica Acervo Saúde, garantindo a ética em pesquisa e os preceitos legais e morais.

A seleção baseou-se em critérios de originalidade, aplicação no âmbito científico, objetividade, clareza, coerência metodológica, apresentação dos dados de forma pertinente e didática, escrita científica e citações atuais e confiáveis. Os 10 trabalhos com melhores pontuações foram selecionados para apresentação na data do evento e concorreram a premiações e menção honrosa.

\section{ANDRÉ RODRIGUES CARVALHO} Diretor Científico do II SIMREABESP 


\section{Resumos Simples}

RESUMO SIMPLES: Revisão Bibliográfica

Título: Consumo alimentar em praticantes de Crossfit: Uma revisão integrativa

Autor/coautores: Gisele Viana Moura, Mateus Cunha de Sousa, Palloma de Sousa, Andrea Nunes Mendes de Brito.

Instituição: Faculdade Estácio de Sá, Teresina, Piauí.

Palavras-chave: Consumo alimentar, Desempenho esportivo, Deficiências Nutricionais.

\section{INTRODUÇÃO}

O Crossfit é uma modalidade de educação física que gera estímulos que promove uma melhora da capacidade física em todo o corpo. Em uma sessão de treino percebem-se resultados positivos, principalmente na composição corporal, pois são utilizadas as vias aeróbias e anaeróbias para produzir energia. Observou-se que uma alimentação equilibrada é necessária para fornecer nutrientes de acordo com as necessidades individuais, frequência e intensidade do treino (RESCANSIN BM, et al., 2019).

\section{OBJETIVO}

Verificar e identificar na literatura a ingestão alimentar em praticantes de Crossfit, bem como identificar possíveis carências nutricionais de vitaminas, minerais e macronutrientes nesses praticantes, e mostrar as evidências cientificas a respeito do assunto.

\section{MÉTODO}

Trata-se de uma revisão integrativa sobre o consumo alimentar em atletas de Crossfit. Realizou-se a busca nas bases eletrônicas Pubmed e SciELO, em agosto de 2020. Descritores e combinações utilizadas: "ingestão alimentar e Crossfit" (food intake and crossfit). Os critérios para a escolha foram: artigos originais, publicados nos últimos cinco anos, em português e inglês, referentes à temática. Foram utilizados 2 artigos na revisão bibliográfica e os estudos que não comtemplaram os critérios foram descartados.

\section{REVISÃO BIBLIOGRÁFICA}

Em uma pesquisa foi avaliado o perfil dietético de 10 homens praticantes de Crossfit por, no minimo, seis meses, e notou-se consumo de dieta hiperproteica e hipolipídica. Quanto aos micronutrientes, os participantes estavam inadequados para todos os micronutrientes. Verificou-se uma baixa ingestão de vitamina $C$, vitamina E, vitamina $K$, Cálcio e Magnésio, e acima para as recomendações diárias de vitamina B6, vitamina B12, Ferro e o Zinco (BUENO BA, et al., 2016).

Em outro estudo realizado com 15 pessoas, na cidade de Mauá - SP, verificou-se que a média de consumo calórico dos participantes foi de 2.126 calorias, e apresentou uma ingestão dos macronutrientes inadequados, mostrando um baixo consumo de carboidratos e alto consumo de proteínas e lipídeos. Além disso, $40 \%$ dos participantes faziam uso de suplementos alimentares, sendo eles: BCAA, beta alanina, cafeína, cromo, creatina, caseinato de cálcio, maltodextrina, whey protein, termogênicos, pré-treinos com taurina, vitaminas, e minerais como zinco e magnésio (ALVES RHV, et al., 2019). 


\section{CONSIDERAÇÕES FINAIS}

Sobre o exposto, verificou-se que os atletas praticantes de Crossfit possuem um consumo alimentar com alto teor de proteínas e gordura, mas com uma ingestão baixa de carboidratos. Além de apresentar possíveis carências de vitaminas lipossolúveis, hidrossolúvel e minerais essenciais para manutenção e rendimento esportivo.

\section{REFERÊNCIAS}

1. ALVES RHV, et al. OBSERVAÇÃO DO CONSUMO INADEQUADO EM ADULTOS PRATICANTES RECREATIVOS DE CROSSFIT DE UMA ACADEMIA EM MAUÁ-SP. UNILUS Ensino e Pesquisa, 2019; 16(44), 76-81;

2. BUENO BA, et al. Determinação da ingestão de micro e macro nutrientes na dieta de praticantes de crossfit. RBNE - Revista Brasileira de Nutrição Esportiva, 2016; 10(59), 579-586.

3. RESCANSIN BM, et al. Análise do perfil alimentar de praticantes de CrossFit na região de Belém do Pará. RBNE-Revista Brasileira De Nutrição Esportiva, 2019; 13(81), 830-838. 
RESUMO SIMPLES: Revisão Bibliográfica

Título: Etiopatogenia da morte súbita cardíaca em jovens atletas

Autor/coautores: André Rodrigues Carvalho, Danyele Holanda da Silva, Izabelle Macedo de Sousa.

Instituição: Faculdade UNINASSAU, Teresina, Piauí

Palavras-chave: Morte súbita cardíaca, Atletas, Etiologia.

\section{INTRODUÇÃO}

Os distúrbios cardiovasculares são as principais causas de morte súbita em jovens atletas durante esportes e exercícios. No entanto, até $80 \%$ das vítimas são assintomáticas antes do início do evento cardiovascular e os distúrbios cardiovasculares preexistentes permanecem não detectados por triagem médica (PETERSON DF, et al., 2020). O conhecimento preciso das causas da morte súbita cardíaca (MSC) em atletas e seus fatores precipitantes como etnia, gênero, diagnóstico cardíaco e esporte, é necessário para implementação de um método eficaz e prático de triagem pré-participação e prevenção (FINOCCHIARO G, et al., 2016).

\section{OBJETIVO}

Identificar na literatura atual as principais causas de MSC em jovens atletas e os fatores que podem predispor ao aumento de sua incidência, como etnia, gênero, diagnóstico cardíaco e o tipo de esporte.

\section{MÉTODO}

Revisão integrativa conduzida de modo a identificar, analisar e sintetizar resultados de estudos independentes sobre a temática já abordada. A busca foi realizada em agosto de 2020 nas bases de dados PubMed, SciELO e LILACS através do cruzamento das palavras-chave morte súbita cardíaca, atletas, etiologia. Foram incluídos artigos publicados entre os anos de 2010 a 2020 nas línguas portuguesa e inglesa que investigaram a etiopatogenia da MSC em jovens atletas. Critérios de exclusão: artigos incompletos, duplicados entre as bases de dados, revisões de literatura, recomendações e diretrizes, intenções de pesquisa e estudos de caso.

\section{REVISÃO BIBLIOGRÁFICA}

Foram identificados 144 artigos destes, sete foram incluídos na revisão. A cardiomiopatia hipertrófica (CMH) tem sido apontada como a principal causa de MSC em atletas (FINOCCHIARO G, et al., 2016). Um estudo observacional realizado nos Estados Unidos constatou que $36 \%$ das MSC em atletas estavam relacionadas à $\mathrm{CMH}$ e 19\% à má formação das artérias coronarianas (MARON BJ, et al., 2016).

O risco de MSC em jovens atletas tem relação direta com algumas variáveis demográficas. Uma busca por dados de MSC em um registro sueco de medicina forense identificou que $90 \%$ dos óbitos ocorreram em indivíduos do sexo masculino (WISTEN A, et al. 2019). Com relação à etnia os afrodescendentes e caucasianos são os mais suscetíveis (HARMON KG, et al., 2016) e a incidência aumenta durante a prática de futebol e basquete (PETERSON DF, et al., 2020).

\section{CONSIDERAÇÕES FINAIS}

Os estudos incluídos na revisão evidenciaram que a MSC em jovens atletas ocorre predominantemente no sexo masculino durante a prática de futebol e basquete. As principais causas de óbito identificadas foram cardiomiopatia hipertrófica e má formação das artérias coronarianas. 


\section{REFERÊNCIAS}

1. FINOCCHIARO G, et al. Etiology of sudden death in sports insights from a united kingdom regional registry. Journal of the american college of cardiology, 2016; 67(18): 2108-2115.

2. HARMON KG, et al. Incidence and etiology of sudden cardiac arrest and death in high school athletes in the united states. Mayo Clin Proc, 2016; 91(11): 1493-1502.

3. MARON BJ, et al. Demographics and epidemiology of sudden deaths in young competitive athletes: from the U.S. National Registry. The American Journal of Medicine, 2016; 129(11): 1170-1177.

4. PETERSON DF, et al. Etiology of sudden cardiac arrest and death in us competitive athletes: a 2-year prospective surveillance study. Clin J Sport Med, 2020; 30(4): 305-314.

5. WISTEN A, et al. Exercise related sudden cardiac death (SCD) in the young - Pre-mortal characterization of a Swedish nationwide cohort, showing a decline in SCD among athletes. Resuscitation, 2019; 144: 99105. 
RESUMO SIMPLES: Revisão Bibliográfica

Título: Influência da pandemia do COVID-19 sobre o ganho de peso da população: Uma revisão integrativa

Autor/coautores: Gisele Viana Moura, Andressa Kelly Lima do Nascimento, Mateus Cunha de Sousa, Carlos Henrique Ribeiro Lima.

Instituição: Universidade Estácio de Sá, Teresina, Piauí.

Palavras-chave: Ganho de Peso, Pandemia, Infecções por Coronavírus.

\section{INTRODUÇÃO}

Mais da metade da população brasileira sofre com excesso de peso, cerca de $56,7 \%$. Com o cenário de pandemia do COVID-19 a prevalência de obesidade ficou bastante preocupante, pois o excesso de peso aumenta os fatores de risco. A obesidade por ser uma doença pró-inflamatória, aumenta o agravamento em pacientes com dificuldade respiratória decorrente pela infeção do COVID-19, existem inúmeros fatores que podem contribuir com o aumento do peso da população durante uma pandemia e um deles é a mudança de rotina adquirida nesse novo contexto mundial (FIGUEIREDO MC, et al., 2020).

\section{OBJETIVO}

Realizar uma revisão integrativa sobre a influência da pandemia no ganho de peso e no comportamento alimentar, bem como expor os achados científicos sobre os fatores contribuintes para o aumento do peso em meio ao surto de pandemia COVID-19.

\section{MÉTODO}

Trata-se de uma revisão integrativa sobre a influência da pandemia covid-19 no ganho de peso. Realizouse a busca nas bases eletrônicas Pubmed e Lilacs, em agosto de 2020. Descritores e combinações utilizadas: "ganho de peso e pandemia" (weight gain AND pandemic) e "obesidade e COVID-19" (obesity and covid-19). Os critérios para a escolha foram: artigos originais, publicados nos últimos cinco anos, em português e inglês, referentes à temática. Foram descartados estudos que não contemplassem os critérios predefinidos. Totalizando em 5 artigos utilizados para a revisão.

\section{REVISÃO BIBLIOGRÁFICA}

Em 2 meses de pandemia em torno de 22\% dos adultos relataram ter ganho de 2,3 a $5 \mathrm{~kg}$ durante a pandemia de COVID-19, os fatores que ocasionaram o surgimento de um novo peso se deu pela diminuição da atividade física, mais lanches noturnos, estresse, baixa ingestão de frutas, irregularidade do sono, alta ingestão de bebida alcoólica ou pela alta ingestão de alimentos ultra processados. Os adultos entrevistados relataram que a ingestão de alimentos quando se estava entediado aumentou muito, cerca de $21,38 \%$ (ZACHARY Z, et al., 2020).

As mudanças no comportamento alimentar e de exercícios físico desde o surgimento da pandemia modificaram muito, em pessoas que já possuía transtornos alimentares foram encontrados aumento dos comportamentos de restrição, compulsão alimentar e exercícios, em pessoas sem transtornos alimentares foi identificado que a realização de exercícios em relação a antes da pandemia diminuiu consideravelmente (PHILLIPOU A, et al., 2020).

\section{CONSIDERAÇÕES FINAIS}


Os estudos investigados demonstram que são inúmeros os fatores de risco que influenciam negativamente no ganho de peso durante a pandemia, tendo como consequências inclusive transtornos alimentares. Faz-se assim necessário o acompanhamento e monitoramento para prevenção de maiores decorrências futuramente.

\section{REFERÊNCIAS}

1. FIGUEIREDO MC, et al. The impact of overweight on clinical complications caused by COVID-19: A systematic review. Research, Society and Development, 2020; 9(7), e693974791.

2. PHILLIPOU A, et al. Eating and exercise behaviors in eating disorders and the general population during the COVID-19 pandemic in Australia: Initial results from the COLLATE project. Int $\mathrm{J}$ Eat Disord. 2020; 53(7), 1158-1165.

3. ZACHARY Z, et al. Self-quarantine and weight gain related risk factors during the COVID-19 pandemic. Obes Res Clin Pract. 2020; 14(3), 210-216. 
RESUMO SIMPLES: Revisão Bibliográfica

Título: O treinamento de equilíbrio como estratégia de controle postural em atletas com instabilidade crônica do tornozelo

Autor/coautores: Bárbara Leite da Silva ${ }^{1}$; Danyele Holanda da Silva²; Rita de Cássia Mendes de Sousa²; Janaína de Moraes Silva'.

Instituição: ${ }^{1}$ Universidade Estadual do Piauí (UESPI), Teresina-Piauí; ${ }^{2}$ Faculdade Maurício de Nassau(UNINASSAU), Teresina-Piauí.

Palavras-chave: Equilíbrio, Postura, Tornozelo.

\section{INTRODUÇÃO}

As entorses do tornozelo são as lesões musculoesqueléticas mais prevalentes nos esportes, sendo capazes de desenvolverem problemas à longo prazo nos atletas, como a instabilidade crônica do tornozelo, a diminuição dos níveis de atividade física e a redução da qualidade de vida (GRIBBLE PA, et al., 2016). As entorses de tornozelo recorrentes podem comprometer o controle postural, podendo levar ao aparecimento de recidivas, fazendo necessário a realização de treinos de equilíbrio para que o corpo permaneça no posicionamento desejado durante a realização de atividades físicas (DONOVAN L, et al., 2016).

\section{OBJETIVO}

Analisar, através da realização de um levantamento na literatura disponível, a eficácia do uso do treinamento de equilíbrio como estratégia de controle postural em atletas com instabilidade crônica do tornozelo.

\section{MÉTODO}

Trata-se de uma revisão integrativa que busca contribuir para a aplicabilidade na prática, sintetizando as pesquisas disponíveis sobre a temática abordada. Realizou-se um levantamento nas bases de dados: Lilacs, Pubmed e Scielo, utilizando os descritores: Postural Balance AND Ankle. Foram incluídos ensaios clínicos randomizados e estudos prospectivos, em inglês e português, originais e publicados de 2015 a 2020, e excluídos artigos duplicados e os que não retratavam o tema proposto.

\section{REVISÃO BIBLIOGRÁFICA}

Foram encontrados 191 artigos, destes, seis foram selecionados. Para a avaliar a instabilidade do tornozelo, foi utilizado o Questionário de Identificação de Instabilidade Funcional do Tornozelo e o equilíbrio foi avaliado pelo Biodex Balance System, Star Excursion Balance Test (SEBT) e Balance Error Scoring System (BESS).

Os exercícios realizados foram os de salto para estabilização, atividades de apoio com um único membro com os olhos abertos e com um único membro com os olhos fechados. O treinamento de equilíbrio consistiu em 18 sessões de 20 minutos e com acompanhamento de 6 semanas após a intervenção. Os resultados obtidos foram a melhora do equilíbrio dinâmico nas direções anterior, posterolateral e posteromedial baseado no SEBT e do equilíbrio estático nas posturas de perna dupla e unilateral com base no BESS.

Os resultados foram alcançados em virtude da recuperação eficiente do controle postural após uma perturbação e desenvolvimento de estratégias para executar metas de movimento (HALL EA, et al., 2018). 


\section{CONSIDERAÇÕES FINAIS}

Conclui-se que o treinamento de equilíbrio é eficaz como estratégia de controle postural em atletas com instabilidade crônica do tornozelo, o que resulta na prevenção de novas entorses do tornozelo por proporcionar o posicionamento adequado do corpo para a realização das atividades físicas.

\section{REFERÊNCIAS}

1. DONOVAN L, et al. Rehabilitation for Chronic Ankle Instability With or Without Destabilization Devices: A Randomized Controlled Trial. J Athl Train, 2016 Mar; 51(3): 233-51.

2. GRIBBLE PA, et al. 2016 consensus statement of the International Ankle Consortium: prevalence, impact and long-term consequences of lateral ankle sprains. British Journal of Sports Medicine, 2016; 50:14931495.

3. HALL EA, et al. Balance- and Strength-Training Protocols to Improve Chronic Ankle Instability Deficits, Part I: Assessing Clinical Outcome Measures. J Athl Train, 2018 Jun; 53 (6): 568-577. 
RESUMO SIMPLES: Revisão Bibliográfica

Título: Suporte nutricional em atletas lesionados: Uma Revisão Integrativa

Autor/coautores: Gisele Viana Moura ${ }^{1}$, Mateus Cunha de Sousa ${ }^{1}$, Carlos Henrique Ribeiro Lima ${ }^{1}$ Matheus Willian Ribeiro de Sousa².

Instituição: Faculdade Estácio de Sá, Teresina , Piauí. Centro Universitário Santo Agostinho, Teresina, Piauí

Palavras-chave: Lesões em Atletas, Suplementação nutricional, Reabilitação.

\section{INTRODUÇÃO}

Em todas as idades tem-se a importância de manter um estilo de vida saudável, devido a vários fatores influenciadores de práticas desportivas, como: relaxamento, competitividade e melhora do estado de saúde. Ainda que o exercício traga benefícios a saúde, ele pode estar associado a lesões esportivas. A estratégia nutricional objetiva-se suprir as necessidades dos atletas, preferencialmente por meio da alimentação, e quando houver necessidade, a suplementação, desta maneira a nutrição vem sendo cada vez mais reconhecida com um componente chave para atuação na recuperação e reabilitação de lesões (MALINAUSKIENE V e MALINAUSKAS R, 2018).

\section{OBJETIVO}

Investigar na literatura a efetividade do suporte nutricional em atletas lesionados, discorrer sobre os benefícios e importância do suporte nutricional nesses atletas, mostrar as evidências científicas e comparar os resultados com a recuperação nessas lesões.

\section{MÉTODO}

Revisão integrativa, pois, possui aplicabilidade dos resultados na prática do suporte nutricional em atletas lesionados pois. Realizou-se a busca nas bases eletrônicas Pubmed e Scielo, em agosto de 2020. Descritores e combinações utilizadas: "athlete feeding and injury" Alimentação de atletas e lesão. Os critérios para a escolha foram: artigos originais, publicados nos últimos cinco anos, em português e inglês, referentes à temática. Foram descartados estudos que não contemplassem os critérios predefinidos.

\section{REVISÃO BIBLIOGRÁFICA}

Atletas nadadores na qual possuía lesão por uso excessivo do tendão, receberam suplementação de creatina e placebo como parte do tratamento conservador da tendinopatia. Grupo da creatina tiveram diminuição da dor significativamente mais rápida durante o tratamento, o pico de toque de flexão plantar do tornozelo possuiu aumento significativo medido no grupo da creatina. Demonstrando que a suplementação de Creatina combinada com estratégia terapêutica apoiou efetivamente a reabilitação da lesão do tendão em nadadores adolescentes (JUHASZ I, et al., 2018).

Estudo avaliou a deficiência de vitamina $D$ e a associação com prejuízo no desempenho muscular em atletas com lesão na medula espinhal, esses foram suplementados com $6.000 \mathrm{UI}$ de vitamina $\mathrm{D}$ diariamente durante 12 semanas, todos os atletas tiveram o status de vitamina $D$ aumentado, atingiram nível ideal para essa vitamina e a força do dinamômetro isocinético foi significativamente aumentada (FLUECK JL, et al., 2016).

\section{CONSIDERAÇÕES FINAIS}

Sobre o exposto, verificou-se que o suporte nutricional combinado com estratégia terapêutica auxilia nas deficiências e necessidades nutricionais advindas das exigências físicas desses atletas, as estratégias 
alimentares poderão ser utilizadas para apoiar a reabilitação da lesão, dessa forma, o suporte nutricional vindo da suplementação e de correções nutricionais contribui efetivamente na recuperação, na qualidade de vida e na promoção de saúde nesses indivíduos.

\section{REFERÊNCIAS}

1. FLUECK JL., et al. Effect of 12-Week Vitamin D Supplementation on 25[OH]D Status and Performance in Athletes with a Spinal Cord Injury. Nutrients. 2016; 8(10): 586.

2. JUHASZ I, et al. Creatine Supplementation Supports the Rehabilitation of Adolescent Fin Swimmers in Tendon Overuse Injury Cases. J Sports Sci Med. 2018; 17(2): 279-288.

3. MALINAUSKIENE V, MALINAUSKAS R. Lifetime Traumatic Experiences and Disordered Eating among University Students: The Role of Posttraumatic Stress Symptoms. Biomed Res Int. 9814358, 2018. 
RESUMO SIMPLES: Revisão Bibliográfica

Título: Efeitos da Eletroestimulação de Corpo Inteiro no metabolismo, na composição corporal e no desempenho físico

Autor/coautores: Daniel dos Santos Nunes, Samuel Nunes Morais, Kelson Nonato Gomes da Silva.

Instituição: Universidade Estadual do Piauí (UESPI), Teresina-PI.

Palavras-chave: Eletroestimulação, Metabolismo, Atividade Física.

\section{INTRODUÇÃO}

A Eletroestimulação de Corpo Inteiro ou WB-EMS (do inglês, whole-body Electromyostimulation) é um método de treinamento relativamente recente, cujo objetivo é potencializar os resultados do treinamento físico em um menor período de tempo. Um colete com eletrodos é utilizado para estimular a contração de grupos musculares simultaneamente a uma rotina de exercícios (PANO-RODRIGUEZ A, et al., 2019). Há evidências de que a técnica oferece benefícios cardiometabólicos, contudo alguns artigos sugerem que os efeitos da WB-EMS são pouco significativos quando comparados a outros tipos de treino. Os resultados dos ensaios que avaliaram a técnica são contraditórios, tornando sua eficácia incerta e a investigação dos seus efeitos relevantes (AMARO-GAHETE FJ, et al., 2020).

\section{OBJETIVO}

Analisar, através de revisão da literatura mais recente, e sintetizar os achados das evidências científicas a respeito dos efeitos da whole-body Electromyostimulation (WB-EMS) no metabolismo, na composição corporal e desempenho físico.

\section{MÉTODO}

Uma revisão integrativa foi feita através de busca por artigos nas bases de dados BVS e PubMED, sendo utilizados descritores em língua inglesa, obedecendo a seguinte estratégia de busca: Whole-body electromyostimulation OR Global body electromyostimulation. Incluíram-se ensaios clínicos controlados publicados entre 2010 e 2020, disponíveis na íntegra, que abordassem os efeitos da WB-EMS. Excluíram-se artigos duplicados, incompletos, revisões e nos quais a eletroestimulação ocorria apenas localmente.

\section{REVISÃO BIBLIOGRÁFICA}

A busca resultou em 26 artigos que atendiam aos critérios estabelecidos. A última busca ocorreu em março de 2020. O uso da WB-EMS como recurso terapêutico se mostrou relevante, demonstrando ter relação com o aumento do percentual de massa corpórea magra, incremento nos processos de oxidação de gorduras durante o exercício físico, melhora na mineralização óssea nos participantes dos estudos e melhora do desempenho em testes funcionais, achados possivelmente justificados pelo incremento metabólico imediato evidenciado e as repercussões do treinamento na aptidão física dos voluntários. Observou-se redução do risco cardiometabólico em populações sedentárias e portadoras de comorbidades, porém, quando avaliada em populações moderadamente treinadas, a WB-EMS não demonstrou superioridade. A análise da WB-EMS em populações idosas e sedentárias foi frequente, havendo poucos estudos que avaliam o recurso em indivíduos jovens ou em praticantes regulares de atividade física (DÖRMANN U, et al., 2019).

\section{CONSIDERAÇÕES FINAIS}

Os resultados demonstram participação positiva da WB-EMS nos parâmetros metabólicos, na proporção entre massa muscular e gordura corporal total, reforçando seu papel na redução do risco cardiometabólico. 
A heterogeneidade dos ensaios torna inviável concluir se os resultados encontrados são válidos para diferentes populações.

\section{REFERÊNCIAS}

1. AMARO-GAHETE FJ, et al. Metabolic rate in sedentary adults, following different exercise training interventions: The fit-ageing randomized controlled trial. Clinical Nutrition, 2020; S0261-5614(20)300492.

2. DÖRMANN U, et al. The effects of superimposed whole-body electromyostimulation during short-term strength training on physical fitness in physically active females: a randomized controlled trial. Frontiers in Physiology, 2019; 10: 728.

3. PANO-RODRIGUEZ A, et al. Effects of whole-body electromyostimulation on health and performance: a systematic review. BMC Complementary and Alternative Medicine, 2019; 19(1): 87. 
RESUMO SIMPLES: Revisão Bibliográfica

Título: Incidência de lesões musculoesqueléticas em praticantes de crossfit: revisão sistemática

Autor/coautores: Talyta da Silva Guimarães ${ }^{1}$, Jederson Valentim Silva², Ariane Luz Carvalho³

Instituição: Faculdade de Educação São Francisco (FAESF), Pedreiras-Maranhão1,2,3.

Palavras-chave: Treinamento intervalado de alta intensidade; Lesões; Crossfit.

\section{INTRODUÇÃO}

O Crossfit é uma modalidade dos exercícios intervalados de alta intensidade, foi criado no início dos anos 2000 e rapidamente popularizou-se por promover aptidão física a partir do desenvolvimento de componentes como capacidade aeróbia, força e resistência muscular, coordenação, velocidade, agilidade e equilíbrio, através da realização de exercícios esportivos e funcionais em altas intensidades (TIBANA RA, et al., 2018). As lesões são problemas que assolam os atletas, e são atribuídas principalmente a realização incorreta dos exercícios que, em geral, está relacionada à complexidade e variação constante dos movimentos (BATTERSON AM, et al., 2019).

\section{OBJETIVO}

Revisar a literatura científica de modo a identificar a prevalência de lesões musculoesqueléticas em praticantes de crossfit, os principais tipos dessas lesões, sua incidência e os fatores de risco associados a estas.

\section{MÉTODO}

Este trabalho consiste em uma revisão sistemática de literatura. Foram utilizadas publicações das principais bases de dados com os descritores: Treinamento intervalado de alta intensidade; Lesões; Crossfit. Os critérios de inclusão foram: publicações dos últimos 5 anos exceto revisões literárias, que analisassem a incidência e/ou a prevalência de lesões musculoesqueléticas em praticantes de Crossfit. Com o cruzamento das palavras-chave foram encontrados 41 artigos, porém após uma filtragem com o protocolo prisma apenas 4 foram utilizados.

\section{REVISÃO BIBLIOGRÁFICA}

Os resultados obtidos em um estudo de coorte demonstraram a incidência de uma taxa de 9 lesões por 1000 horas de treinamento em exercícios de alta intensidade, o que foi aproximadamente o triplo das lesões encontradas na literatura para este tipo de exercício (BATTERSON AM, et al., 2019). Já em um estudo de avaliação de prontuários de uma clínica esportiva, houve a prevalência de lesões em indivíduos do sexo feminino, que em sua maioria ocorreram nos membros inferiores (SUGIMOTO D, et al., 2019). Uma pesquisa observacional retrospectiva constatou que $39,9 \%$ dos entrevistados através de um questionário relataram lesões musculoesqueléticas após o início dos treinos de Crossfit (TAFURI et al., 2018). Em uma análise de 270 praticantes de Crossfit foi observado que $22,6 \%$ dos indivíduos sofreram lesões sendo estas em sua maioria articulares e localizadas no ombro e coluna lombar (MINGHELLI B e VICENTE B, 2019).

\section{CONSIDERAÇÕES FINAIS}

Com a análise dos artigos foi possível identificar que a modalidade Crossfit tem um potencial lesivo. Porém, os resultados entre os artigos foram controversos, limitados e restritos e que por tanto faz-se necessário a produção de mais pesquisas na área. 


\section{REFERÊNCIAS}

1. BATTERSON, AM et al. Injury Rate and Patterns in Group Strength-Endurance Training Classes. Mayo Clin Proc. 2019; 95(3):468-475

2. MINGHELLI B, VICENTE P. Lesões musculoesqueléticas em praticantes portugueses de CrossFit. J Sports Med Phys Fitness 2019; 59: 1213-20.

3. SUGIMOTO, D et al. Part II: Comparison of Crossfit-Related Injury Presenting to Sports Medicine Clinic by Sex and Age. Clin J Sport Med 2019; 00:1-6

4. TAFURI, S et al. The risk of injuries among Crossfit athletes: na italian observational restrospective survey. The Journal of Sports Medicine and Physical Fitness. 2018. 59(9): 1544-1550.

5. TIBANA, RA et al. Relação da força muscular com o desempenho no levantamento olímpico em praticantes de CrossFit@. Rev Andal Med Deporte 2018; 11:84-8. 
RESUMO SIMPLES: Revisão Bibliográfica

Título: Influência da Vitamina D sobre a massa óssea em indivíduos fisicamente ativos

Autor/coautores: Gisele Viana Moura ${ }^{1}$, Rayana Rodrigues da Silva² ${ }^{2}$ Tatiele Castelo de Oliveira², Vanessa Batista de Sousa Lima ${ }^{3}$

Instituição: ${ }_{1}$ Universidade Estácio de Sá, Teresina, Piauí, 2 Centro Universitário Santo Agostinho, Teresina, Piauí; 3Doutora em Ciências dos Alimentos pela Universidade de São Paulo- USP.

Palavras-chave: Vitamina D, Exercício físico, Saúde.

\section{INTRODUÇÃO}

A Vitamina D é um hormônio lipossolúvel, derivada de esteróis que atua principalmente no metabolismo do cálcio e do fósforo. Na presença de raios ultravioleta, a pró-vitamina $D$ é convertida na forma ativa da VitD. Estudos demonstram o importante papel regulador da vitamina $D$ em estruturas ósseas e musculoesqueléticas. Apresenta grande influência na saúde óssea, como reguladora na homeostase do cálcio, fosfato e no metabolismo ósseo, colaborando de forma positiva na eficácia dos resultados e no desempenho em atletas isso se dar pela presença de receptores de vitamina $D$ no tecido muscular. (MENESES PSD, et al., 2018).

\section{OBJETIVO}

Realizar uma revisão integrativa da literatura sobre a vitamina $D$ e analisar como ela pode influenciar na melhoria, no desenvolvimento e no fortalecimento da massa óssea em indivíduos fisicamente ativos.

\section{MÉTODO}

Revisão integrativa realizada em agosto de 2020, nas bases de dados: SciELO e PubMed, utilizando os descritores em português e inglês: "vitamina D e massa óssea" e "vitamina D e exercício físico". Incluiu-se artigos que abordavam a temática em estudo. Excluiu-se os estudos que não se encaixavam na linha de pesquisa, totalizando 5 artigos.

\section{REVISÃO BIBLIOGRÁFICA}

Em um ensaio randomizado realizado com dois grupos de aprendizes de jóqueis do sexo masculino, foram avaliadas propriedades ósseas durante 6 meses de condução. Os resultados mostraram que a vitamina $D$ apresentou alterações satisfatórias no metabolismo ósseo, melhorando os níveis de densidade mineral óssea, sendo capaz de proporcionar maior suporte de peso aos atletas (SILK LN, et al., 2017).

A vitamina $D$ foi associada à potencialização de força muscular isométrica de pernas e braços, bem como na isocinética de braços, constatando que boas concentrações dessa vitamina podem otimizar a melhoria na recuperação da força do músculo esquelético em praticantes de musculação, possibilitando, o aumento da concentração de cálcio e a homeostase do metabolismo ósseo no organismo. Ademais, nesse estudo mostrou certificado ainda, que o consumo de VD para praticantes de atividade física de alta intensidade é fundamental para prevenção de lesões musculares durante o movimento (REZENDE TM e CĖSAR TCM, 2019).

\section{CONSIDERAÇÕES FINAIS}

A vitamina D potencializa o metabolismo ósseo, a densidade óssea e melhora na recuperação da força do musculo em indivíduos treinados. Ademais, apresenta impacto no aumento da concentração de cálcio e na homeostase do metabolismo ósseo no organismo, demonstrando assim, sua influência sobre a realização do exercício físico. 


\section{REFERÊNCIAS}

1. MENESES PSD, et al. Vitamina $D$ na prática esportiva: revisão de escopo. Revista Brasileira de Prescrição e Fisiologia do Exercício, 2018; 12(80): 1093-1100.

2. REZENDE T. M; CEZAR, T. C. M. Conhecimento da Importância do Consumo de Vitamina D por Praticantes de Atividade Física em Academias de Cascavel-PR. Fag journal of health (FJH), 2019; 1(1): 107-118.

3. SILK L.N, et al. The effect of calcium and vitamin D supplementation on bone health of male Jockeys. J Sci Med Sport, 2017; 20(3): 225-229. 
RESUMO SIMPLES: Revisão Bibliográfica

Título: Atuação da fisioterapia no pós-operatório de reconstrução do ligamento cruzado anterior (LCA)

Autor/coautores: Jessé Sales de Barros, Luiz Valdean Sobrinho Nascimento, Mariana Andrade Jaques de Castro, Haértori da Silva Leal.

Instituição: Instituto de Educação Superior Raimundo Sá, Picos- PI - IESRSA.

Palavras-chave: Fisioterapia. Ligamento Cruzado anterior. Reconstrução.

\section{INTRODUÇÃO}

A articulação do joelho é uma das mais complexas articulações do corpo humano. Com isso, é alvo de uma multiplicidade de lesões que modificam o seu desempenho e sua função. A ruptura do ligamento cruzado anterior (LCA) é uma lesão que ocorre de diferentes maneiras, especialmente nos esportes de contato como o futebol, quando há um mecanismo de desaceleração ou uma mudança de direção associado ao giro, com o pé fixo no solo. A alta ocorrência de lesões neste ligamento leva a um aumento de instabilidade do joelho (RÊGO AS, et al., 2014).

\section{OBJETIVO}

Verificar frente a literatura a respeito da atuação da fisioterapia no pós-operatório de reconstrução do Ligamento Cruzado Anterior, suas principais técnicas, assim também como os objetivos destas para ter êxito no tratamento.

\section{MÉTODO}

Trata-se de uma revisão de literatura. A busca de estudos para a síntese foi realizada no Scielo e Google Acadêmico no período de julho de 2020. Os descritores utilizados para a busca foram: "Physiotherapy" "Anterior Cruciate Ligament", "reconstrution". Como critério de seleção foram escolhidos estudos observacionais ou de intervenção, em idiomas português e inglês publicados entre 2014 a 2019, foram excluídas revisões bibliográficas e artigos duplicados. Foram analisados 28 artigos, e após a observação de critérios de elegibilidade, foram escolhidos 6 artigos elegíveis para a síntese.

\section{REVISÃO BIBLIOGRÁFICA}

Não existe um protocolo de reabilitação considerado padrão, o que existe são parâmetros que devem ser seguidos para que o fisioterapeuta tenha base para evoluir com a reabilitação, tratando o paciente no pósoperatório de uma lesão de LCA de forma segura e rápida. (KARIZE STM, 2017). Com isso, o tratamento fisioterapêutico deve reduzir os efeitos adversos da imobilização, sem sobrecarregar os tecidos em fase de cicatrização, permitindo, assim, que o paciente volte a sua capacidade funcional anterior a lesão. (MATHEUS SS, et al., 2016).

Uma maior proporção dos artigos mostraram a atuação eficiente da fisioterapia no pós-operatório de reconstrução de LCA com o uso da crioterapia com o intuito de reduzir dor e melhorar o processo inflamatório, técnicas de mobilização articular para o complexo do joelho, alongamentos, principalmente de posteriores, exercícios ativo-assistido para flexão e extensão de joelho a fim de zerar extensão e melhorar a ADM; exercícios em CCA, contrações isométricas, principalmente de quadríceps para ativá-lo, prevenir hipotrofia; exercícios proprioceptivos para melhorar o equilíbrio, e consequentemente restabelecer a marcha.

\section{CONSIDERAÇÕES FINAIS}


A atuação da Fisioterapia mostrou eficácia nas condições clínicas pós-operatória de reconstrução Ligamento Cruzado Anterior, prevenindo complicações no processo de cicatrização, melhorando o quadro álgico, prevenindo hipotrofia, restaurando a ADM e restabelecendo a marcha. Dessa forma, proporciona uma melhor condição para que o paciente possa retornar a suas práticas esportivas e as atividades da vida diária da melhor forma possível.

\section{REFERÊNCIAS}

1. ADRIANA RS, et al. Atuação da fisioterapia no pré e pós-operatório de reconstrução do ligamento cruzado anterior (LCA). Revista Brasileira de Prescrição e Fisiologia do Exercício, 2014; 8.46.

2. ANTONIO RJM, DANIEL NBM. REABILITAÇÃO FISIOTERÁPICA APÓS RECONSTRUÇÃO DO LIGAMENTO CRUZADO ANTERIOR DO JOELHO. FATECI, 2015.

3. FRANCISCO AES. ATUAÇÃO DA FISIOTERAPIA NO PÓS-OPERATÓRIO DE LIGAMENTO CRUZADO ANTERIOR." PITÁGORAS, 2019; 41(14).

4. KARIZE STM. Fisioterapia em lesão de ligamento cruzado anterior com ênfase no tratamento pósoperatório. Fisioterapia Brasil, 2017; 12(1); 47-52.

5. MAITÉ P, et al. Tratamento fisioterapêutico após reconstrução do ligamento cruzado anterior. Acta Ortopédica Brasileira, 2015; 372-375.

6. MATHEUS SS, et al. INTERVENÇÃO FISIOTERAPÊUTICA NO PÓS-OPERATÓRIO DE LESÕES DO LIGAMENTO CRUZADO ANTERIOR. Revista Eletrônica de Ciências, 2016; 11.16. 
RESUMO SIMPLES: Revisão Bibliográfica

Título: Dados antropométricos e ingestão alimentar em atletas de voleibol: Uma revisão Integrativa

Autor/coautores: Mateus Cunha de Sousa, Gisele Viana de Moura, Jerônimo Gregrio Da Silva Neto, Andrea Nunes Mendes de Brito.

Instituição: Faculdade Estácio de Sá, Teresina, Piauí.

Palavras-chave: Voleibol, Avaliação nutricional, Ingestão alimentar.

\section{INTRODUÇÃO}

Os atletas de voleibol necessitam de habilidades técnicas, pois precisam correr, saltar, bloquear e defender. Para isso, o atleta necessita de força e potência muscular, que são importantes para melhorar o desempenho físico. A antropometria e composição corporal são essenciais para determinar o rendimento dos atletas, além de melhorar o desempenho nos treinos e auxiliar significativamente nas competições. Uma alimentação adequada é importante para melhorar o estado nutricional e parâmetros antropométricos dos atletas, além de maximizar o desempenho físico e auxiliar na recuperação e manutenção da saúde dos praticantes dessa modalidade (FACCIN AP, et al., 2017).

\section{OBJETIVO}

Verificar na literatura a importância da antropometria e ingestão alimentar na busca de melhoria do desempenho esportivo de atletas de voleibol, bem como demonstrar o estado nutricional desses atletas de voleibol.

\section{MÉTODO}

Trata-se de uma revisão integrativa sobre a importância da antropometria e ingestão alimentar de atletas de voleibol. Realizou-se buscas na base eletrônica "SciEIO" e "Google acadêmico", em agosto de 2020. Descritores utilizados: "Alimentação e vôlei" e Antropometria e vôlei". Os critérios para inclusão foram: artigos originais, publicados nos últimos três anos (2017-2020), em português, referentes à temática. Foram selecionados 2 artigos científicos.

\section{REVISÃO BIBLIOGRÁFICA}

Pesquisa avaliou atletas que treinavam entre 10 a 12 horas semanais. Os autores dividiram as atletas em (EqSNut) que não possuíam um acompanhamento nutricional e (EqCNut) que possuíam um acompanhamento nutricional cerca de 3 anos. As atletas que tinham um acompanhamento nutricional apresentaram um peso mais adequado em relação a estatura, soma das dobras cutâneas menores, percentual Gordura (\%) inferior, massa magra $(\mathrm{Kg})$ superior e IMC $\left(\mathrm{Kg} / \mathrm{m}^{2}\right)$ mais adequado comparado com as atletas sem acompanhamento nutricional (RIGOBELLO NS e ROTHER RL, 2017).

Estudo composto por atletas do sexo masculino apresentaram um IMC com média de $22,02 \mathrm{~kg} / \mathrm{m}^{2}$, mas cerca de $10 \%$ dos atletas apresentaram magreza grau 1 . O percentual de gordura dos atletas foi em média de $13,10 \%$, estando adequado e dentro da normalidade. Os atletas consumiam média de 3709,39 kcal diárias, considerado hipercalórica para a modalidade e uma ingestão de proteínas, carboidratos e lipídeos dentro do recomendado pelas Dietary Reference Intakes (DRIs) (MELO AM, et al., 2017).

\section{CONSIDERAÇÕES FINAIS}

Os resultados deste estudo levam a conclusão de que o acompanhamento antropométrico e a ingestão alimentar adequada podem enriquecer a avaliação nutricional de atletas de voleibol, já que a avaliação nutricional permite avaliar a composição nutricional e a análise da ingestão alimentar. 


\section{REFERÊNCIAS}

1. FACCIN AP, et al. Perfil antropométrico e alimentar e o conhecimento nutricional de atletas de voleibol. RBNE - Revista Brasileira De Nutrição Esportiva, 2017; 11(63), 259-264.

2. MELO AM, et al. Avaliação do estado nutricional e consumo alimentar de jogadores de voleibol masculino da categoria adulta em uma cidade no interior do estado de São Paulo. RBNE - Revista Brasileira De Nutrição Esportiva, 2017; 11(65), 593-602.

3. RIGOBELLO NS, Rother RL. Comparação do perfil antropométrico de equipes de Voleibol feminino de base: a importância do Nutricionista. RBNE - Revista Brasileira De Nutrição Esportiva, 2017; 11(61), 7480. 
RESUMO SIMPLES: Revisão Bibliográfica

Título: Efeitos da acupuntura em atletas: revisão bibliográfica

Autora/ coatores: Danyele Holanda da Silva ${ }^{1}$; Letícia de Sousa Vidal²; Bárbara Leite da Silva ${ }^{3}$; Rita de Cássia Mendes de Sousa ${ }^{4}$; Janaína de Moraes Silva ${ }^{5}$.

Instituição: Faculdade Maurício de Nassau- Redenção (UNINASSAU), Teresina-PI1,4; Universidade Estadual do Piauí (UESPI), Teresina-PI',3,5

Palavras-chave: Acupuntura, Benefícios, Atletas.

\section{INTRODUÇÃO}

Os atletas em geral estão sempre susceptíveis a ter lesões, tanto os amadores como os profissionais, no entanto, os atletas de elite, quando lesionados tem a necessidade de voltar rapidamente a prática do esporte, pois além dos contratos que ele precisa cumprir, a falta de treinamento pode reduzir o seu rendimento no esporte (LEE TL e MARX BL, 2018). Tendo em vista que a acupuntura é uma medicina que tem crescido exponencialmente ao longo dos anos, e por ser conhecida como um método não invasivo que pode aliviar dores e melhorar sistemas, podendo ser benéfica como terapia auxiliar tanto para analgesia como para recuperação do atleta lesionado (SIQUEIRA APR, et al., 2018).

\section{OBJETIVO}

Investigar na literatura os resultados de estudos selecionados para o resumo, que tinham como desfecho quais eram os efeitos da acupuntura associada a outras intervenções na analgesia e recuperação de atletas lesionados.

\section{MÉTODO}

Revisão de literatura desenvolvida a partir de artigos submetidos a comunidade científica por meio do portal de periódicos: Pubmed, Lilacs e Scielo, através dos descritores "acupuncture in athlets" e "Benefícios da Acupuntura em atletas". Incluídos os artigos completos, nos idiomas inglês e português, datados entre 2015 e 2020, excluídos artigos indisponíveis e que não abordassem o tema. Foram encontrados 134 artigos, sendo quatro elegíveis.

\section{REVISÃO BIBLIOGRÁFICA}

Em três estudos, aplicadas como intervenção em atletas de diferentes modalidades, técnicas de combinação de terapias manuais (massagens, liberação do gatilho miofascial, liberação de alongamento, etc) associado a sessões de acupuntura, tendo como objetivo analisar o efeito da junção destas técnicas na diminuição de dor e também inchaço em diferentes partes do corpo desta população estudada, logo os três estudos constataram diminuição de dor e inchaço (PUGAZHENDI S, et al., 2020).

O quarto estudo realizou a aplicação de dois questionários de dor, a aplicação de um teste de força máxima com avaliação eletromiográfica do reto femoral, vasto lateral e aplicação de acupuntura na região do joelho em atletas com relato de dor nesta região, concluindo que todos os participantes relataram uma diminuição da dor após o tratamento (SIQUEIRA APR, et al., 2018).

\section{CONSIDERAÇÕES FINAIS}

Nota-se os benefícios do uso da acupuntura associada a outras intervenções na diminuição da dor e inchaço e aumento de ganho de força em diferentes regiões do corpo de atletas. Tendo como limitação, poucos estudos com a acupuntura abordada isoladamente. 


\section{REFERÊNCIAS}

1. LEE TL, MARX BL. Noninvasive, Multimodality Approach to Treating Plantar Fasciitis: A Case Study. JAMS Journal of Acupuncture and Meridian Studies, 2018; 11(4): 162-164.

2. PUGAZHENDI S, et al. Non-invasive complementary therapies in managing musculoskeletal pains and in preventing surgery. International Journal of Therapeutic Massage and Bodywork: Research, Education, and Practice, 2020; 13(2): 9-18.

3. SIQUEIRA APR, et al. Reduction in knee pain symptoms in athletes using an acupuncture protocol. Acta Ortopedica Brasileira, 2018; 26(6): 418-422. 
RESUMO SIMPLES: Revisão bibliográfica

Título: Efeitos das meias compressivas em atletas: uma revisão bibliográfica

Autora/ coatores: Danyele Holanda da Silva1; Letícia de Sousa Vidal2; André Rodrigues Carvalho³ Neivaldo Ramos da Silva4; Izabelle Macedo de Sousa ${ }^{5}$

Instituição: Faculdade Maurício de Nassau- Redenção (UNINASSAU), Teresina-PI'1,3,4,5; Universidade Estadual do Piauí (UESPI), Teresina-PI²

Palavras-chave: Meias compressivas, Efeitos, Atletas.

\section{INTRODUÇÃO}

Meias de compressão (MC) são meias que criam uma pressão compressiva ao redor do músculo, osso e tecido conjuntivo, sendo essa pressão maior na região do tornozelo e diminuindo gradativamente até o joelho (MORENO PD, et al., 2020). Os atletas vestem essas roupas com o intuito de adquirirem benefícios fisiológicos e musculares, tais como alteração na distribuição do volume sanguíneo, melhorar o retorno venoso e criar uma hemodinâmica mais favorável dos membros centrais e superiores, entre outros, nestes indivíduos. Logo estes potenciais podem afetar o treinamento, o desempenho e a recuperação pós-exercício (VAILE J, et al., 2016).

\section{OBJETIVO}

Investigar através do levantamento bibliográfico realizado nas bases de dados, os resultados de estudos selecionados para o resumo, que tinham como desfecho avaliar os efeitos das meias compressivas em atletas.

\section{MÉTODO}

Revisão de literatura desenvolvida a partir de artigos submetidos a comunidade científica por meio do portal de periódicos: Pubmed, Scielo, Lilacs, através dos buscadores "compressive socks" AND "athletes" AND "effects", encontrados 138 artigos que abordassem meias compressivas em atletas. Incluídos artigos originais datados entre 2015 e 2020, nos idiomas inglês, português e espanhol, e excluídos artigos incompletos, indisponíveis ou que não abordassem o tema, sendo cinco artigos que se encaixaram nos critérios de inclusão.

\section{REVISÃO BIBLIOGRÁFICA}

Corrida de $1 \mathrm{~km}$ com 16 atletas avaliados as mudanças na atividade eletromiográfica pelas variáveis metabólicas com uso de MC na performance de desempenho destes (MORENO PD, et al., 2020). 20 atletas, em corrida, examinaram o efeito do uso de MC na pressão intramuscular dos músculos da perna e alteração precoce de biomarcadores sanguíneos (RENNERFELT K, et al., 2019). 19 atletas, em corrida de 5km, avaliados respostas fisiológicas e perceptuais ao uso de MC (TRESELER C, et al., 2016). Na pesquisa de VAILE J, et al., 2016, investigaram os efeitos das MC durante o exercício sobre o desempenho e as respostas fisiológicas em 10 atletas, em exercício ao redor de quadra poliesportiva. Avaliados o fluxo sanguíneo da panturrilha e antebraço medido usando extensômetro. Num estudo com 20 atletas, avaliadas as respostas cardiorrespiratórias através das MC durante corrida após três semanas de uso regular (PRIEGO JI, et al., 2015).

\section{CONSIDERAÇÕES FINAIS}

Os benefícios adquiridos em atletas com uso de meias compressivas foram tempo médio da volta, sendo maior e o fluxo sanguíneo do membro superior melhor mantido nos grupos que utilizaram a mesma, porém os malefícios foram relato de aumento da pressão intramuscular e índice de oxigenação tecidual inferior. 


\section{REFERÊNCIAS}

1. MORENO PD, et al. Muscle activation in middle-distance athletes with compression stockings. Sensors (Switzerland), 2020; 20 (5): 1-8.

2. RENNERFELT K, et al. Effects of Exercise Compression Stockings on Anterior Muscle Compartment Pressure and Oxygenation During Running: A Randomized Crossover Trial Conducted in Healthy Recreational Runners. Sports Medicine, 2019; 49(9): 1465-1473.

3. VAILE J, et al. Effect of lower limb compression on blood flow and performance in elite wheelchair rugby athletes. Journal of Spinal Cord Medicine, 2016; 39(2): 206-211. 
RESUMO SIMPLES: Revisão bibliográfica

Título: Uso da eletroestimulação em pós-operatório de cirurgia de reconstrução do ligamento cruzado anterior em atletas: revisão bibliográfica

Autor/coautores: Rita de Cássia Mendes de Sousa ${ }^{1}$, Danyele Holanda da Silva ${ }^{1}$, Bárbara Leite da Silva², Janaína de Moraes Silva².

Instituição: ${ }^{1}$ Faculdade UNINASSAU, Teresina, Piauí; ${ }^{2}$ Universidade Estadual do Piauí (UESPI), Teresina, Piauí

Palavras-chave: Terapia por Estimulação Elétrica, Terapêutica, Ligamento Cruzado Anterior.

\section{INTRODUÇÃO}

Uma das principais lesões de membros inferiores em atletas é a ruptura do Ligamento Cruzado Anterior (LCA), a qual provoca instabilidade na articulação do joelho (ZHANG L, et al., 2018). A cirurgia de reconstrução do LCA é o tratamento mais utilizado, a qual poderá ter certas complicações (dor patelofemoral, atrofia ou fraqueza muscular e etc) (FOROGH B, et al., 2017). No pós-operatório (PO) a eletroestimulação é geralmente utilizada para fortalecimento. As técnicas mais utilizadas são a Estimulação Elétrica Funcional (FES) e a Estimulação Elétrica Neuromuscular (NMES) (WRIGHT AR, et al., 2019).

\section{OBJETIVO}

Analisar na literatura os desfechos encontrados sobre o uso das técnicas FES e NMES para o tratamento de fortalecimento muscular no PO de cirurgia de reconstrução do LCA em atletas.

\section{MÉTODO}

Trata-se de uma revisão integrativa, a qual consiste na elaboração de um estudo da literatura, em prol de investigar sobre um assunto específico, de acordo com o apanhado de várias pesquisas (FERREIRA M, et al., 2019). Esta pesquisa foi composta por artigos sobre o uso de eletroestimulação no PO de cirurgia de reconstrução de LCA, realizada nas plataformas PEDro e PubMed com palavras chaves: Electric Stimulation Therapy AND Therapeutics AND Anterior Cruciate Ligament. Foram incluídos na pesquisa artigos dos últimos 5 anos, e excluídos os que não retratavam sobre o tema.

\section{REVISÃO BIBLIOGRÁFICA}

Foram encontrados 328 artigos e incluídos cinco artigos na pesquisa. No PO da cirurgia de reconstrução do LCA, é comum a fraqueza do músculo quadríceps. Com isso, autores afirmam que a inibição neural pode causar essa falha de ativação do quadríceps (SONNERY-COTTET B, et al., 2019).

Como auxílio no tratamento, tem-se utilizado o FES, indicado para fortalecimento muscular com uso de aprendizagem motora através da prática repetitiva, e o NMES, também indicado para fortalecimento, a qual se pode utilizar uma corrente de baixa ou média frequência. Uma pesquisa realizada com 23 indivíduos, no período de 4 semanas de PO, comprovou que o FES em comparação com o NMES, ambos utilizados juntamente com o tratamento convencional, é mais eficiente no aumento da simetria e força muscular, resultando em melhora da marcha e prevenção de futuras lesões (MORAN U, et al., 2019).

\section{CONSIDERAÇÕES FINAIS}

Analisou-se que a inibição neural pode estar relacionada com a fraqueza do músculo quadríceps no PO de reconstrução do LCA. Com isso, o FES tem destaque para aumento da força e simetria muscular, melhorando a marcha e prevenindo contra novas lesões. 


\section{REFERÊNCIAS}

1. FOROGH B, et al. Adicionar a estimulação elétrica nervosa transcutânea de alta frequência à primeira fase da reabilitação pós-reconstrução do ligamento cruzado anterior não melhora a dor e a função em jovens atletas do sexo masculino mais do que apenas o exercício: um ensaio clínico randomizado simplescego. Disability and Rehabilitation, 2017; 41(5): 514-522.

2. MORAN U, et al. Estimulação elétrica funcional após reconstrução do ligamento cruzado anterior: um estudo piloto randomizado controlado. J Neuroeng Rehabil, 2019; 16(1): 89.

3. SONNERY-COTTET B, et al. Inibição muscular artrogênica após reconstrução do LCA: uma revisão do escopo da eficácia das intervenções. Br J Sports Med, 2019; 53(5): 289-298.

4. WRIGHT AR, et al. Eficácia do desempenho de recuperação acelerada para reabilitação pósreconstrução do LCA. Hawaii J Health Soc Welf, 2019; 78(11): 41-46.

5. ZHANG L, et al. Mecanismo de ativação da via de sinalização proprioceptiva NT-3 / TrkC por intervenção reversa para o arco reflexo do ligamento cruzado anterior-isquiotibiais com eletroacupuntura. BioMed Research International, 2018; 2018: e6348764. 


\section{AGRADECIMENTOS}

\section{Patrocinadores}
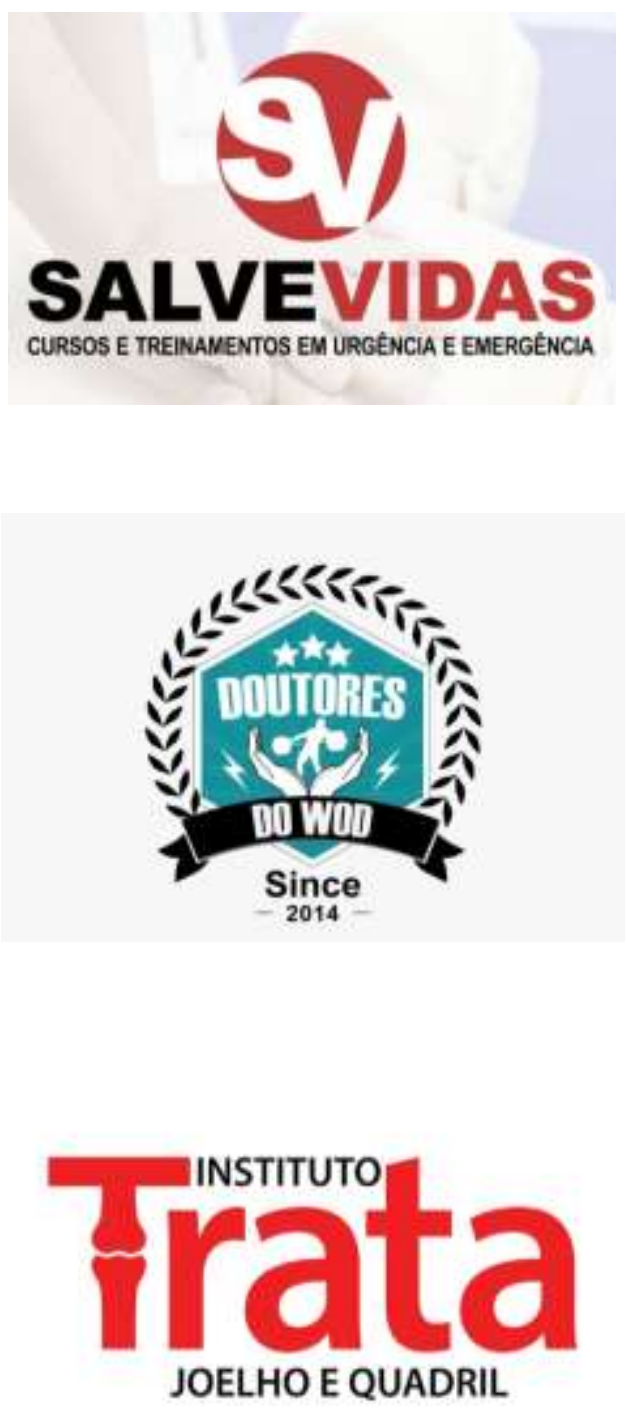